\title{
Perfis metabólico e nutricional como preditores da síndrome da apneia obstrutiva do sono
}

\author{
Metabolic and nutritional status as \\ predictors of obstructive sleep \\ apnea syndrome
}

Marinaldo Freire LUSTOSA ${ }^{1}$

Cristiane Maria Araújo Tavares de SÁ ${ }^{1}$

Adrilene Coutinho CAVALCANTI ${ }^{1}$

Rafael Augusto Batista de MEDEIROS²

Larissa Pessoa Vila NOVA ${ }^{1}$

Rodrigo Pinto PEDROSA ${ }^{1}$

\section{RES U M O}

\section{Objetivo}

Correlacionar os perfis metabólico e nutricional com a presença e gravidade da síndrome da apneia obstrutiva do sono.

\section{Métodos}

Estudo transversal com pacientes adultos e idosos, de ambos os sexos, atendidos no Laboratório do Sono e Coração do Pronto Socorro Cardiológico Universitário de Pernambuco, entre junho e setembro de 2014. Os pacientes se submeteram à polissonografia, à bioimpedância elétrica e a aferições antropométricas. Foram verificadas a presença da síndrome metabólica e outras morbidades.

\section{Resultados}

A amostra total foi constituída por 50 pacientes, com idade média de 57,52 $\pm 9,80$ anos, sendo que 94\% receberam diagnóstico de síndrome da apneia obstrutiva do sono e 74\% possuíam síndrome metabólica. As seguintes médias foram obtidas: índice de massa corporal $\left(31,54 \pm 5,82 \mathrm{~kg} / \mathrm{m}^{2}\right)$; circunferência do pescoço $(39,14 \pm 4,33 \mathrm{~cm})$; circunferência da cintura $(106,72 \pm 11,22 \mathrm{~cm})$; diâmetro abdominal sagital $(23,00 \mathrm{~cm}$ [21,00-24,00]). O índice de massa corporal, a circunferência da cintura e o diâmetro abdominal sagital apresentaram valores

\footnotetext{
1 Universidade de Pernambuco, Programa de Residência em Nutrição Clínica. Pronto-Socorro Cardiológico de Pernambuco. Av. Agamenon Magalhães, s/n., Santo Amaro, 50100-010, Recife, PE, Brasil. Correspondência para/Correspondence to: MF LUSTOSA. E-mail: <marinaldofreire@yahoo.com.br>.

${ }^{2}$ Universidade Federal Rural de Pernambuco, Pró-Reitoria de Gestão Estudantil e Inclusão, Coordenadoria de Gestão de Alimentação e Restaurante Universitário, Recife, PE, Brasil.
} 
mais elevados $(p<0,05)$ entre os pacientes com síndrome da apneia obstrutiva do sono grave, quando comparados aqueles com a forma leve. O diâmetro abdominal sagital apresentou moderada correlação com o índice de apneia e hipopneia e a gordura corporal apresentou fraca correlação.

\section{Conclusão}

A obesidade, a circunferência do pescoço, o diâmetro abdominal sagital e a síndrome metabólica tiveram associação positiva com a gravidade da síndrome da apneia obstrutiva do sono. Dentre os parâmetros antropométricos avaliados, o diâmetro abdominal sagital mostrou ser o mais adequado preditor para avaliar a presença e gravidade da síndrome da apneia obstrutiva do sono.

Palavras-chave: Antropometria. Apneia do sono tipo obstrutiva. Bioimpedância elétrica. Diâmetro abdominal sagital.

\section{A B S T R A C T}

\section{Objective}

To compare metabolic and nutritional profiles with the presence and severity of obstructive sleep apnea syndrome.

\section{Methods}

This cross-sectional study included male and female adults and older adults treated at the Sleep and Heart Laboratory of Pernambuco Cardiologic Emergency Medical Services between June and September 2014. Patients underwent polysomnography, bioelectrical impedance analysis, and anthropometric measurements. Presence of the metabolic syndrome and other morbidities was investigated.

\section{Results}

The sample consisted of 50 patients with a mean age of $57.52 \pm 9.80$ years, of which $94 \%$ were diagnosed with obstructive sleep apnea syndrome, and $74 \%$ had the metabolic syndrome. Other mean sample characteristics were: body mass index $\left(31.54 \pm 5.82 \mathrm{~kg} / \mathrm{m}^{2}\right)$; neck circumference $(39.14 \pm 4.33 \mathrm{~cm})$; waist circumference $(106.72 \pm 22.11 \mathrm{~cm})$; sagittal abdominal diameter $(23.00 \mathrm{~cm}$ [21.00-24.00]). Patients with severe obstructive sleep apnea syndrome had higher body mass index, waist circumference, and sagittal abdominal diameter $(p<0.05)$ than those with mild condition. Sagittal abdominal diameter was correlated moderately with the apnea-hypopnea index and mildly with body fat.

\section{Conclusion}

Obesity, neck circumference, and the metabolic syndrome had a positive association with obstructive sleep apnea syndrome severity. Of the study anthropometric parameters, sagittal abdominal diameter was the most suitable predictor of presence and severity of obstructive sleep apnea syndrome.

Keywords: Anthropometry. Apnea, obstructive sleep. Bioelectrical impedance analysis. Sagittal abdominal diameter.

\section{N T R O D U Ç Ã O}

A Síndrome da Apneia Obstrutiva do Sono (SAOS) é uma doença crônica caracterizada por episódios parciais ou completos de colapso das vias aéreas superiores associados ao sono intermitente, que resulta na dessaturação de oxigênio e fragmentação do sono. Os sintomas incluem desde o ronco até a sonolência diurna, bem como consequências metabólicas fatais. A síndrome da apneia obstrutiva do sono é classificada de acordo com a frequência de apneia e hipopneia do sono: leve $(5-14,9 / \mathrm{h})$, moderada $(15-29 / \mathrm{h})$ e grave $(\geq 30 / h)^{1-3}$.
O excesso de peso é conhecido como importante fator de risco para síndrome, embora também possa ocorrer em indivíduos magros. A obesidade geral e, principalmente, a abdominal contribuem como principais fatores determinantes de hipoventilação e, além disso, colaboram para o aparecimento da apneia obstrutiva que aumenta o colapso das vias aéreas superiores ${ }^{4-6}$. Portanto, devido à grande importância da avaliação da gordura abdominal, torna-se necessário realizar a antropometria para a sua detecção.

Dentre as variáveis antropométricas, destaca-se a Circunferência da Cintura (CC), a Cir- 
cunferência Abdominal (CA) e o Diâmetro Abdominal Sagital (DAS). Este último, por sua vez, é conhecido como a altura abdominal. É um dos métodos menos difundidos na prática clínica, contudo tem sido cada vez mais utilizado para determinar a extensão da adiposidade visceral ${ }^{7,8}$.

A maioria das evidências existentes reflete uma forte correlação entre o percentual de gordura corporal (estimado pelas medidas antropométricas e bioimpedância) e a gravidade da SAOS 5 . Lee \& Gallagher ${ }^{9}$ observaram que o percentual de gordura corporal estimado por bioimpedância previu o risco de SAOS, de forma a contribuir para a probabilidade de pré-teste (probabilidade estimada da doença antes do teste ser realizado).

A presença da SAOS resulta em alterações metabólicas e distúrbios nos sistemas endócrino, imune e inflamatório, de forma a aumentar o risco para Hipertensão Arterial Sistêmica (HAS), Infarto Agudo do Miocárdio (IAM) e Síndrome Metabólica (SM). Esta é composta por um grupo de comorbidades composto por aumento da pressão arterial, elevação dos Triglicerídeos (TG), do Colesterol Total (CT), da lipoproteína de baixa densidade e da Glicose de Jejum (GJ), bem como pela diminuição dos níveis da lipoproteína de alta densidade. Em pacientes com SM, a prevalência tende a ser maior em relação à SAOS moderada a severa ${ }^{10,11}$. Nessa população, a SAOS está independentemente associada ao aumento da glicose de jejum e dos TG, assim como a maiores níveis de marcadores inflamatórios, arteriosclerose e aterosclerose ${ }^{12}$.

Estudos epidemiológicos mostram que a prevalência de SAOS associada com sonolência diurna é aproximadamente de 3 a $7 \%$ para homens adultos e de 2 a $5 \%$ para mulheres adul$\operatorname{tas}^{2-4}$. Particularmente na HAS, estudos mostram uma prevalência de síndrome da apneia obstrutiva do sono de $35 \%$, chegando a $70 \%$ em casos de hipertensão arterial refratária. A prevalência da patologia atinge cerca de 40 e $70 \%$ dos pacientes com obesidade e sobrepeso, respectivamente ${ }^{13,14}$. Nesse contexto, partindo-se do pressuposto de que a obesidade, sobretudo na região abdominal, pode estar fortemente associada à SAOS, o objetivo do trabalho foi correlacionar os perfis metabólico e nutricional com a presença e gravidade da síndrome da apneia obstrutiva do sono.

\section{M É T O D O S}

Trata-se de um estudo transversal em que foram avaliados pacientes com idade igual ou maior que 20 anos, de ambos os sexos, atendidos no Laboratório do Sono e Coração do Pronto Socorro Cardiológico Universitário de Pernambuco Professor Luiz Tavares, no período de junho a setembro de 2014. Foram incluídos os pacientes com diagnóstico ou não de doenças crônicas, como diabetes Mellitus, dislipidemia, HAS, obesidade e coronariopatias. Foram excluídos aqueles indivíduos com sinais de edema, ascite ou anasarca, mulheres gestantes, indivíduos com incapacidade de se manter de pé e aqueles com hepato ou esplenomegalia. Informações sobre idade (em anos completos), sexo, situação conjugal (casados/união estável e não casados), renda familiar (categorizada em 1 a 2, 3 a 6 e >6 salários mínimos) e grau de escolaridade (ensino fundamental, médio e superior) também foram coletadas.

As variáveis utilizadas na avaliação do estilo de vida foram: nível de atividade física, tabagismo e etilismo. A primeira foi analisada de acordo com os critérios do American College of Sports Medicine ${ }^{15}$. Já o alcoolismo foi considerado quando o paciente referia ingerir quantidade de bebida alcoólica $>30 \mathrm{~g} /$ dia, para o sexo masculino, e $>15 \mathrm{~g} /$ dia para o feminino ${ }^{16}$. Foi considerado fumante o indivíduo que consumiu cigarro no ano anterior à inclusão no estudo; não fumante aquele que nunca fumou ou que não fumava há mais de 10 anos; e ex-fumante o que não fumou no ano anterior ao estudo, mas fumou entre um e dez anos antes ${ }^{17}$.

Os pacientes foram pesados em posição ereta, com roupas leves e descalços, em balança digital tipo plataforma da marca Filizola ${ }^{\mathrm{TM}}$ (FilizolaTM ${ }^{\mathrm{TM}}$ São Paulo, SP, Brasil), capacidade máxi- 
ma de $180 \mathrm{Kg}$ e variação de 100 gramas. A altura foi verificada através de estadiômetro metálico da marca Tonelli® ${ }^{\circledR}$ Tonelli® ${ }^{\circledR}$ Criciúma, Santa Catarina, Brasil), fixado à parede com altura máxima de 2,2 metros e frações de $1 \mathrm{~mm}$. Os pacientes se mantiveram em posição ereta, com os calcanhares juntos, costas retas e membros superiores pendentes ao longo do corpo ${ }^{18}$. Em indivíduos idosos, a altura foi obtida pela altura do joelho, através da fórmula de Chumlea et al. ${ }^{19}$, a qual foi aferida com régua antropométrica com escala $\mathrm{em} \mathrm{mm}^{20}$. A obesidade foi classificada através do Índice de Massa Corporal (IMC) e seus pontos de corte preconizados pela World Health Organization ${ }^{17}$, para adultos, e pela Organización Panamericana de la Salud ${ }^{21}$, para idosos.

Com o objetivo de identificar o padrão de distribuição da massa adiposa (obesidade abdominal), foram utilizados os índices: circunferência da cintura, diâmetro abdominal sagital e índice de conicidade. A circunferência da cintura foi aferida com uma fita métrica não extensível no ponto médio entre a última costela e a crista ilíaca, de modo que a leitura foi realizada no momento da expiração. A obesidade abdominal foi definida pelos pontos de corte estabelecidos pela World Health Organization ${ }^{17}$.

O diâmetro abdominal sagital foi avaliado com o indivíduo em posição supina, com os joelhos inclinados sobre uma superfície plana e firme. A aferição foi feita sob a roupa, após uma expiração normal, na distância entre o dorso em contato com a superfície e o ponto mais elevado do abdômen, entre a última costela e a crista ilíaca. O equipamento utilizado foi um paquímetro de metal com extensão de $50 \mathrm{~cm}$ (Cescorf ${ }^{\circledR}$, São Paulo, SP, Brasil) $)^{21}$.

Já o índice de conicidade foi determinado através das medidas de peso, estatura e circunferência da cintura, seguindo a seguinte equação matemática ${ }^{22}$ :

$$
I C=\frac{\text { Circunferência da cintura }(\mathrm{m})}{0,109 \sqrt{\begin{array}{c}
\text { Peso corporal }(\mathrm{kg}) / \\
\text { Estatura }(\mathrm{m})
\end{array}}}
$$

A Circunferência do Pescoço (CP) foi medida com o auxílio de uma fita métrica da marca Sanny ${ }^{\circledR}\left(\right.$ Sanny ${ }^{\circledR}$, São Bernardo do Campo, São Paulo, Brasil). Os participantes ficaram eretos, com a cabeça posicionada no plano horizontal de Frankfort. A borda superior da fita métrica foi colocada logo abaixo da proeminência da laringe e aplicada perpendicularmente ao longo do eixo do pescoço ${ }^{23}$. Para a análise do percentual de Gordura Corporal (\%GC), foi utilizada a Bioimpedância Elétrica, quantificada através de equipamento portátil da marca Biodynamics modelo 310 (Biodynamics, Morumbi, São Paulo, Brasil), que aplica uma corrente de $800 \mu \mathrm{A}$, com frequência simples de $50 \mathrm{kHz}$.

A presença das seguintes comorbidades foi verificada por intermédio do prontuário médico: diabetes Mellitus, HAS e dislipidemia, que podiam também ser autorreferidas ou subentendidas pelo uso de medicações específicas, como anti-hipertensivos, hipolipemiantes e hipoglicemiantes orais. A presença da síndrome metabólica também foi avaliada segundo os critérios da "V Diretriz Brasileira de Dislipidemias e Prevenção da Aterosclerose" 24 .

O Índice de Apneia e Hipopneia (IAH) foi obtido através de exame polissonográfico, por meio da divisão do total de eventos respiratórios pelas horas de sono. Os pacientes foram classificados de acordo com o IAH em: sem apneia menos de 5,0 eventos/hora de sono; com apneia leve - entre 5,0 e 14,9 eventos/hora de sono; com apneia moderada - entre 15,0 e 30,0 eventos por/hora de sono; e com apneia grave - mais de 30,0 eventos/hora de sono ${ }^{25}$.

Para as coletas de sangue, os pacientes permaneceram em jejum por pelo menos 12 horas. As recomendações da " $\vee$ Diretriz de Dislipidemia e Prevenção de Aterosclerose" 24 também foram utilizadas como padrão para avaliação do perfil lipídico, tendo sido avaliados O CT e lipoproteína de alta densidade, dosados pelo método calorimétrico-enzimático homogêneo com sensibilidade de $0,009 \mathrm{mmol} / \mathrm{L}(0,3 \mathrm{mg} / \mathrm{d} / \mathrm{L})$, $0,004 \mathrm{mmol} / \mathrm{L}(1,55 \mathrm{mg} / \mathrm{dL})$ e $0,10 \mathrm{mmol} / \mathrm{L}$ 
(3,87 mg/L), respectivamente, com leitura através de aparelho COBAS Íntegra 400 Plus (Roche Diagnostics, São Paulo, SP, Brasil). O lipoproteína de baixa densidade foi obtido através da fórmula de Friedewald ${ }^{26}$. O triglicerídeos foi determinado pelo método calorímetro-enzimático (GPO/PAP, Glycerol Phosphate Oxidase/Peroxidase) com glicerol fosfato oxidase e 4-aminofenazona e sensibilidade de $0,04 \mathrm{mmol} / \mathrm{L}(3,5 \mathrm{mg} / \mathrm{d} / \mathrm{L})$. A glicemia de jejum foi classificada segundo as "Diretrizes da Sociedade Brasileira de Diabetes" 27 e determinada pelo método enzimático de referência com hexoquinase e sensibilidade de $3,5 \mathrm{mmol} / \mathrm{L}$ (63 mg/d/L).

O Protocolo do estudo foi aprovado pelo Comitê de Ética em Pesquisa envolvendo seres humanos do Complexo Hospitalar da Universidade de Pernambuco (Hospital Universitário Oswaldo Cruz e Pronto-Socorro Cardiológico Universitário de Pernambuco), de acordo com a Resolução $n^{\circ} 466 / 12$ do Conselho Nacional de Saúde, sob o n $641.761 / 2014$. Os pacientes que concordaram em participar da pesquisa assinaram o Termo de Consentimento Livre e Esclarecido após receberem todas as informações do estudo.

Para o teste estatístico, foi realizada a análise exploratória dos dados (exclusão dos outliers). As variáveis contínuas foram testadas quanto à normalidade da distribuição através do teste de Kolmogorov Smirnov e aplicadas transformações logarítimicas ( $\left.\log _{\text {neperiano }}\right)$, quando necessárias. Os dados das variáveis de distribuição normal foram expressos na forma de média e desvio-padrão. Para as variáveis que apresentaram normalidade apenas após a transformação logarítmica, os dados foram expressos na forma de média geométrica e seus respectivos intervalos de confiança. Aquelas com distribuição não Gaussiana foram apresentadas sob a forma de medianas e dos respectivos intervalos interquartílicos. O teste Wilcoxon Soma-de-Ranks (Mann-Whitney) foi usado para comparação de duas amostras não compensadas. Os dados foram digitados em dupla entrada e verificados com o Validate, módulo do Programa Epi Info, versão 6.04 (Epi Info,
Clifton Road Atlanta, GA, Estados Unidos da América), para checar a consistência e validação das informações. Já a análise estatística foi realizada com o auxílio do programa Statistical Package for Social Sciences, versão 13.0 (SPSS, Chicago, Illinois, Estados Unidos da América).

\section{RES U L T A D O S}

No total, foram recrutados 72 indivíduos e apenas um foi excluído por possuir edema e marca-passo. Houve uma perda na amostra de 21 pacientes por motivos como a rejeição da participação e o não comparecimento no dia marcado. A amostra final foi composta por 50 pacientes, com idade média de 57,52 $(57,52 \pm 9,80)$ anos. O sexo feminino $(58 \%)$, a pequena renda familiar ( $58 \%$ ) e a baixa escolaridade (58\%) foram predominantes.

Observou-se que mais da metade eram indivíduos sedentários (64,0\%), não fumantes $(80,0 \%)$ e não etilistas $(68,0 \%)$. Em relação às características clínicas e metabólicas, verificou-se elevada prevalência de HAS $(90,0 \%)$ e SM $(74,0 \%)$ enquanto que diabetes Mellitus apresentou prevalência de $36 \%$. Diagnosticou-se a SAOS em grande parcela da amostra (94,0\%), sobretudo entre as mulheres $(55,3 \%)$.

Em relação ao perfil nutricional, foi verificada maior prevalência de obesos $(60,0 \%)$, seguida de uma taxa de $24,0 \%$ de indivíduos com sobrepeso e $16,0 \%$ de eutróficos. A respeito daqueles que foram diagnosticados com SAOS, $85,1 \%$ apresentaram excesso de peso, sendo que $59,6 \%$ estavam obesos. As características dos pacientes e sua distribuição de acordo com a presença e gravidade da SAOS podem ser encontradas na Tabela 1.

Em relação à $C C$, não houve diferença quanto à significância estatística entre os grupos avaliados, entretanto foi observada uma tendência crescente no grupo SAOS leve quando comparado ao grupo sem SAOS. O peso, o IMC, a CC e a CP foram diretamente proporcionais à gravidade da SAOS, de forma que o grupo SAOS 


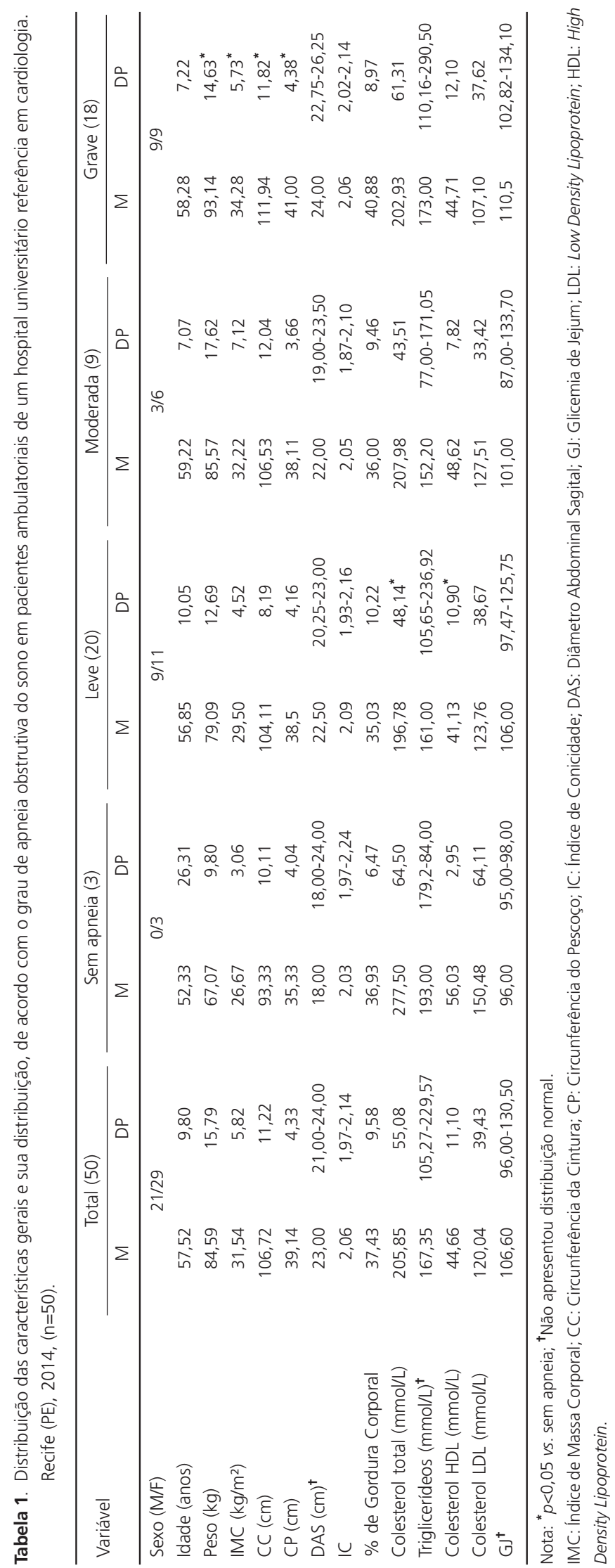


grave apresentou diferença quanto à significância estatística $(p<0,05)$ em relação ao grupo sem SAOS. No tocante ao perfil lipídico, os valores de CT $(p<0,05)$ e lipoproteína de alta densidade $(p<0,05)$ apresentaram uma relação inversa com a gravidade da SAOS. A relação entre SAOS leve, moderada e grave é ilustrada na Figura 1.
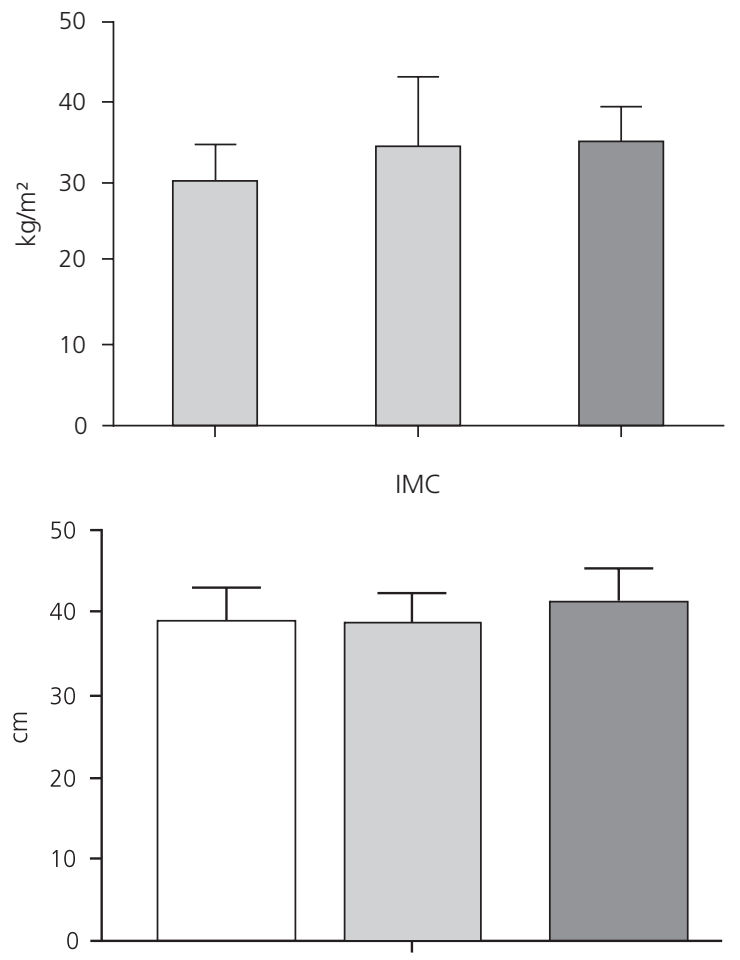

CP

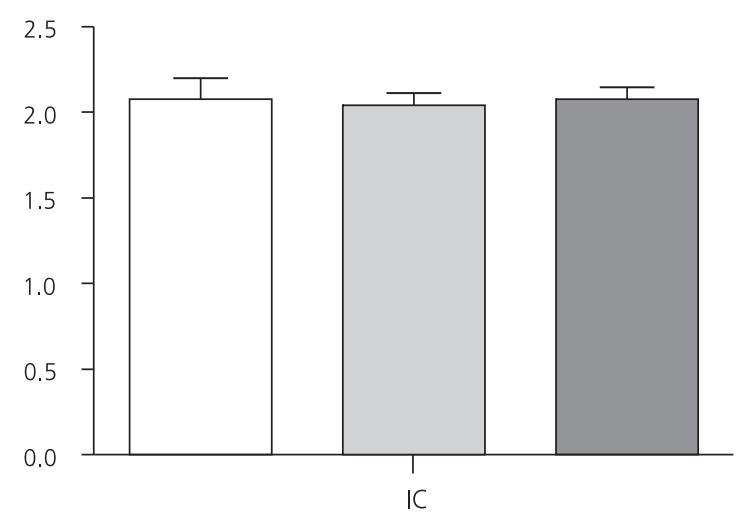

Dentre as variáveis estudadas, apenas o DAS exibiu significância estatística no grupo SAOS grave, quando comparado ao grupo SAOS moderada $(p<0,05)$. O índice de massa corporal, a CC e o DAS apresentaram valores mais elevados $(p<0,05)$ entre os pacientes com SAOS grave do que naqueles com SAOS leve. A correlação entre
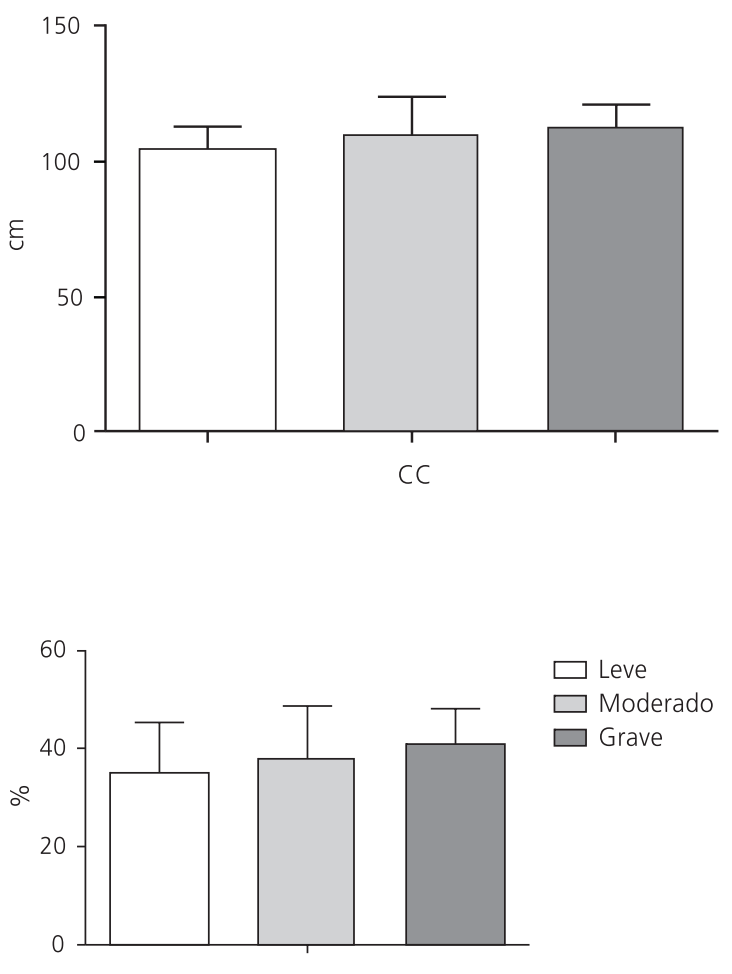

GC

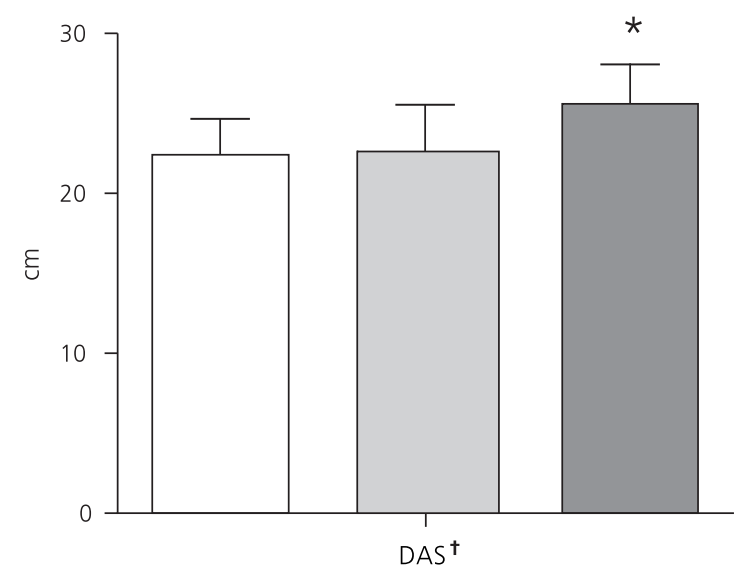

Figura 1. Relação entre os diferentes graus de apneia de acordo com os parâmetros de avaliação nutricional ${ }^{*}$ de pacientes ambulatoriais de um hospital universitário referência em cardiologia. Recife (PE), $2014(n=50)$.

Nota: *Teste t-student; ${ }^{\dagger}$ Não apresentou distribuição normal: Teste de "U" Mann-Whitney.

IMC: Índice de Massa Corporal; CC: Circunferência da Cintura; CP: Circunferência do Pescoço; GC: Gordura Corporal; IC: Índice de Conicidade; DAS: Diâmetro Abdominal Sagital. 

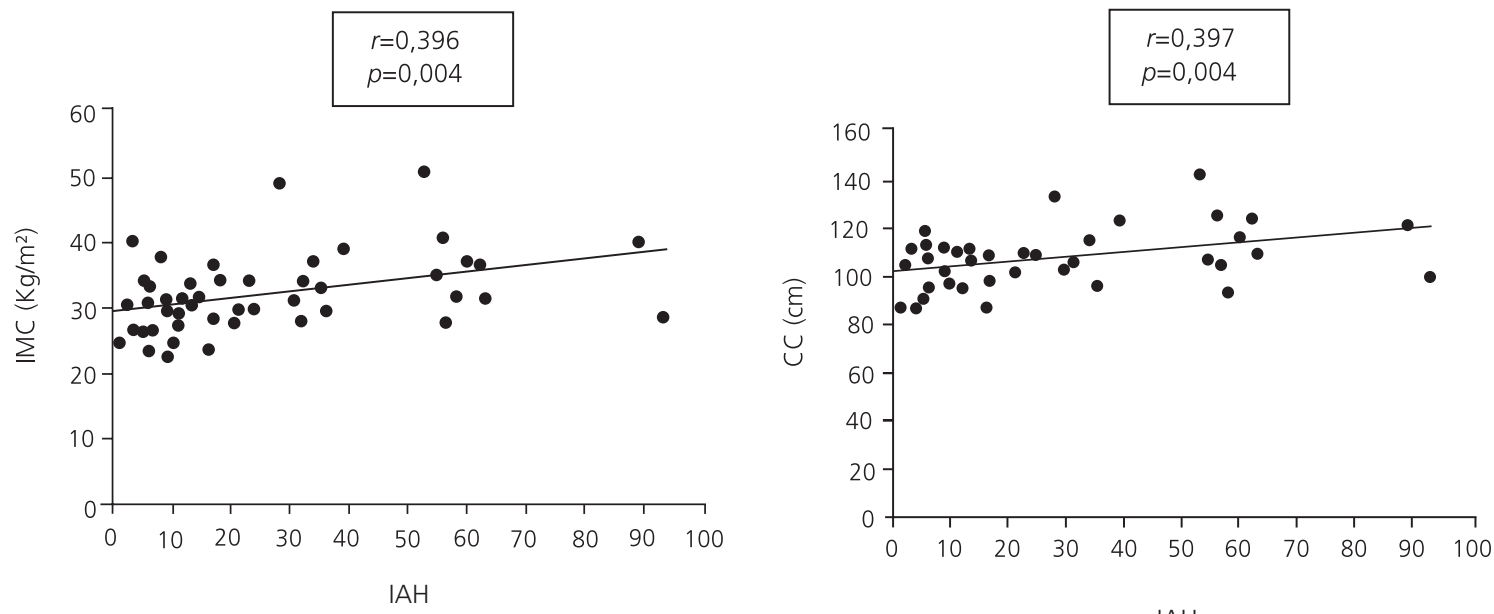

$\mathrm{IAH}$
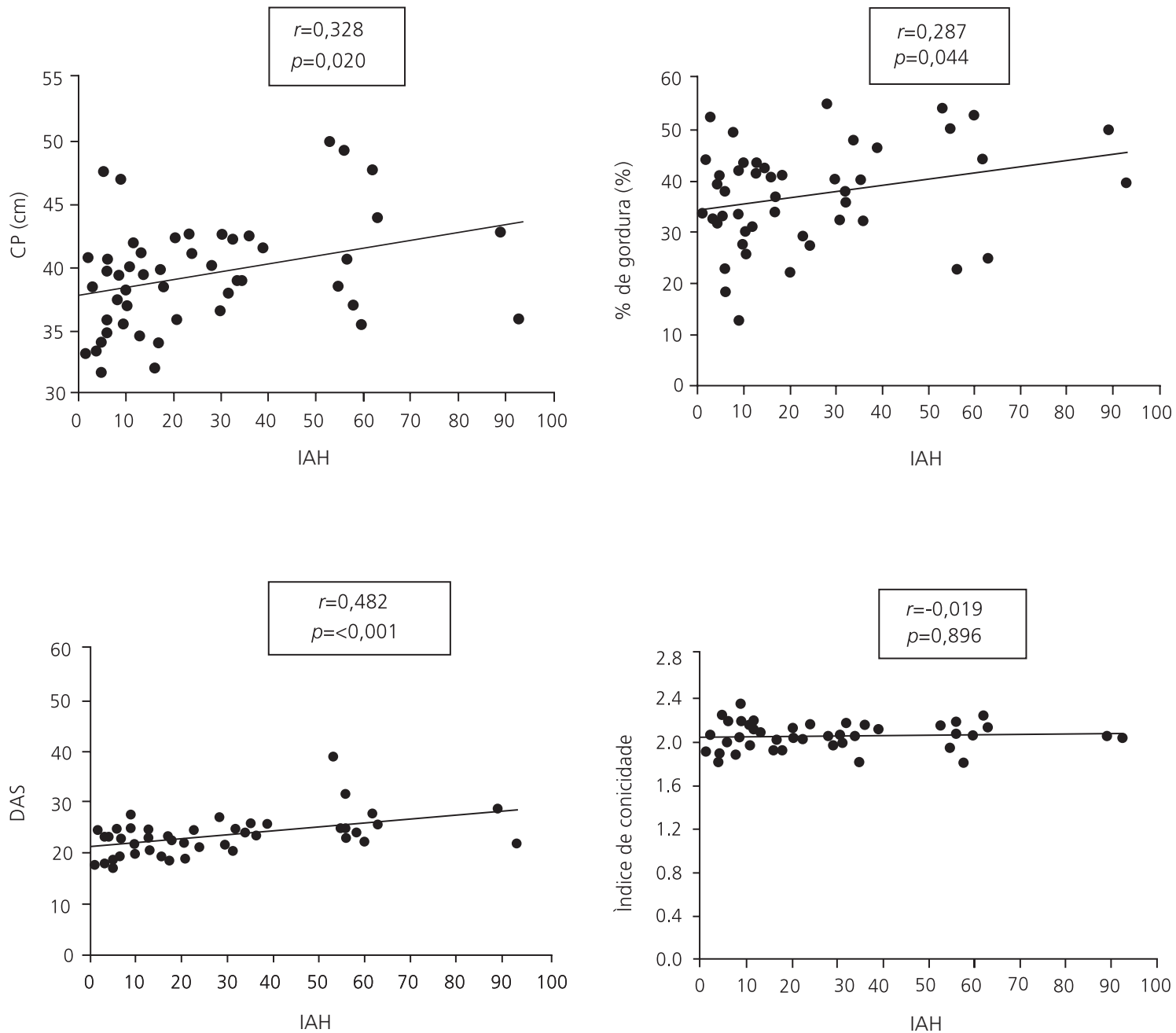

Figura 2. Correlação entre o Índice de Apneia e hipopneia (IAH) e variáveis de avaliação nutricional de pacientes ambulatoriais de um hospital universitário referência em cardiologia. Recife (PE), 2014 ( $n=50)$.

Nota: IMC: Índice de Massa Corporal; CC: Circunferência da Cintura; CP: Circunferência do Pescoço; DAS: Diâmetro Abdominal Sagital; IAH: Índice de Apneia e Hipopneia. 
o IAH e o IMC, a CC, a CP e o \% GC foi fraca, sendo que apenas a correlação entre o IAH e o DAS mostrou-se moderada. O índice de conicidade não apresentou nenhuma correlação com O IAH (Figura 2).

\section{I S C U S S Ã O}

A faixa etária média no estudo foi maior que 50 anos e a maior parte dos participantes apresentou HAS. Na pesquisa de Franklin et al. ${ }^{28}$, o maior grau de apneia foi associado à idade avançada, uma vez que a apneia do sono pode atingir o pico em torno de 55 anos de idade, particularmente em homens. Em parte, a HAS se deve ao aumento da pressão arterial sistólica isolada em idosos quando comparados a indivíduos jovens, além de ser um preditor válido para eventos cardiovasculares nessa faixa etária. Existe uma correlação positiva entre idade, obesidade e circunferência do pescoço. Dessa maneira, à medida que a idade avança, o peso corpóreo e o comprimento da circunferência do pescoço, que são fatores de risco para a apneia, tendem a aumentar 29

Nesta pesquisa, a SAOS foi mais prevalente entre as mulheres. Todavia, Young et al..$^{30}$, através de estudo epidemiológico realizado nos Estados Unidos da América, encontrou maior prevalência no sexo masculino. Essa divergência pode ser justificada pela maior busca das mulheres pelo serviço de saúde, visto que o presente trabalho foi conduzido com pacientes encaminhados para a investigação da SAOS. Outros estudos epidemiológicos ${ }^{31}$ corroboraram as maiores prevalências em homens de diagnóstico e gravidade da SAOS, com proporções na faixa de 1,6:1 e 2:1 a $3: 1^{2,32}$. Chang \& Wang ${ }^{31}$ confirmaram a maior gravidade da SAOS em homens, embora o IMC nas mulheres fosse maior. Diferenças entre os sexos em relação à SAOS foram elucidadas de acordo com os seguintes postulados: 1) o maior comprimento da via aérea faríngea e do palato mole em homens resulta na maior capacidade de deformação das vias aéreas ${ }^{33}$; 2) a distribuição da gor- dura em homens é predominantemente central, incluindo gordura visceral e gordura corporal superior, que pode atuar como importante fator de risco para SAOS ${ }^{34}$; 3) redução de hormônios femininos no pós menopausa em mulheres francesas afetou a distribuição de gordura corporal e foi observado um significativo aumento na proporção de gordura androide e na taxa de gordura do tronco/gordura das pernas, o que aumentou o risco e gravidade da SAOS ${ }^{35}$; 4) hormônios femininos, como a progesterona, são considerados estimulantes respiratórios, de forma a proteger as mulheres contra eventos nas vias aéreas superiores $^{36}$.

Quanto ao perfil nutricional, a SAOS mostrou-se mais frequente em indivíduos com excesso de peso (85\%), o que corrobora os achados de Shimura et al. ${ }^{29}$ que investigou 185 pacientes com $70 \%$ de sobrepeso em portadores da SAOS. O excesso de peso e a obesidade são fatores de risco para desenvolvimento da SAOS em ambos os sexos e resultam em maiores níveis de leptina circulante, indicando que essa condição está relacionada a um estado de resistência à leptina causada por defeitos em receptores ou pós-receptores ${ }^{31}$.

Além disso, há evidências crescentes de que a regulação da leptina é alterada na SAOS. Estudos anteriores mostraram que os níveis circulantes de leptina estão positivamente correlacionados aos índices de adiposidade e à gravidade da SAOS. Os níveis mais elevados de leptina podem contribuir para a deposição de gordura preferencial (subcutânea vs. visceral), a qual pode predispor o desenvolvimento da síndrome. Polotsky et al. ${ }^{37}$ sugerem que o tratamento da SAOS através da perda de peso poderia sensibilizar os tecidos periféricos no que diz respeito à leptina, podendo reduzir a deposição de gordura ao redor da faringe e diminuir a carga mecânica nessa região. Isso, consequentemente, resultaria na melhora do controle neuromuscular.

As concentrações de leptina em pacientes com SAOS podem contribuir para a fisiopatologia da doença de várias formas. Demonstrou-se, em 
estudos com animais, que a hiperleptinemia crônica poderia promover a elevação da pressão sanguínea, agregação de plaquetas e trombose arterial. Essas observações levantam a possibilidade de que a leptina pode ter um papel no desenvolvimento da hipertensão e da doença cardiovascular em pacientes com SAOS ${ }^{38}$. Segundo Drager et al. ${ }^{12}$, a hipóxia no tecido adiposo decorrente da síndrome da apneia obstrutiva do sono pode representar um mecanismo importante das disfunções cardiovasculares e metabólicas, porque é um disparador principal da lipólise, da inflamação crônica, da infiltração de macrófagos, da redução do nível de adiponectina, da elevação do nível de leptina e da disfunção mitocondrial.

Sobre o estilo de vida, verificou-se pequeno percentual de etilistas e tabagistas. Por outro lado, Bhama et al. ${ }^{39}$ encontrou correlação positiva entre a SAOS e o tabagismo e consumo excessivo de álcool. Dessa maneira, os dados encontrados neste trabalho divergem da literatura. Entretanto, os resultados aqui apresentados podem ser explicados pelo fato da ingestão aguda de álcool produz relaxamento nos músculos responsáveis pela permeabilidade da via aérea e altera a respiração durante o sono. Já quanto aos tabagistas, a redução da concentração de nicotina sanguínea nos fumantes poderia levar a uma instabilidade durante o sono, fator que predispõe a obstrução das vias aéreas superiores ${ }^{40}$.

Pacientes com diagnóstico de SAOS apresentaram maior prevalência de SM, entretanto não houve diferença de acordo com o grau da SAOS. Lubrano et al. ${ }^{6}$ também encontraram um percentual de SM maior entre os pacientes com a síndrome $(65,4 \%)$, porém, diferente desta pesquisa, a prevalência da SM estava aumentada em função da gravidade da SAOS: $52,4 \%$ dos pacientes com SAOS leve, $69,2 \%$ dos com SAOS moderada e 77,8\% dos com SAOS grave. Embora a relação da SAOS com a SM tenha sido amplamente atribuída à obesidade, os mecanismos exatos da associação entre a síndrome e suas características individuais ainda permanecem não elucidados ${ }^{14}$. Entretanto, Frija-Orvoën ${ }^{11}$ sugere que esse resultado seja provavelmente devido à hipóxia intermitente da SAOS.

No presente estudo, a gravidade da SAOS mostrou uma associação mais forte com a CC, IMC e CP. Kritikou et al. ${ }^{34}$, através de tomografia computadorizada, relataram que a adiposidade visceral é o principal fator associado à SAOS em homens, enquanto que a adiposidade geral é mais notada em mulheres. Os resultados deste estudo sugerem que a adiposidade abdominal e a total podem prever a gravidade da SAOS melhor do que a composição corporal pela bioimpedância elétrica. Embora a coleta de dados tenha sido padronizada, alguns fatores que limitam o uso da bioimpedância elétrica devem ser considerados, como estado de hidratação, posição corporal, prática de exercícios físicos, ingestão dietética, temperatura da pele e ciclo menstrual, pois podem afetar diretamente os resultados encontrados $^{41}$. Além disso, o equipamento de bioimpedância utilizado no estudo avaliou a gordura total, sem compartimentalização dos estoques de gordura corporal. O percentual de gordura, apesar de ter sido maior na SAOS grave, não apresentou associação com significância estatística. A possível causa seria o tamanho da amostra, principalmente do grupo sem a SAOS. Por outro lado, Punjabi relatou aumento da gravidade da SAOS com aumento da idade e IMC. De forma concordante com o presente estudo, Hoffstein \& Mateika ${ }^{42}$ observaram que os pacientes com apneia tiveram maiores valores de IMC e CP que aqueles sem apneia. Neste estudo, a média do IMC e CP foi de $32,3 \pm 7,6 \mathrm{~kg} / \mathrm{m}^{2}$ e $42,7 \pm 4,4 \mathrm{~cm}$, respectivamente, no grupo apneico, ao passo que a média de IMC e CP foi de $28 \pm 5,3 \mathrm{~kg} / \mathrm{m}^{2}$ e $38,4 \pm 3,9 \mathrm{~cm}$ no grupo sem apneia.

No tocante à glicemia de jejum, maiores valores foram encontrados nos grupos com SAOS grave. Polotsky et al. ${ }^{43}$, ao estudar ratos obesos submetidos à hipóxia intermitente em curto prazo, observaram uma diminuição nos níveis de glicose no sangue acompanhada por um aumento nos níveis séricos de insulina, a qual foi abolida por infusão de leptina anteriormente. Os ratos obesos 
expostos a intermitentes hipóxias durante 12 semanas (longo prazo) desenvolveram um aumento dependente do tempo de jejum nos níveis séricos de insulina e a piora da tolerância à glicose, o que indica a resistência à insulina. Os autores concluíram que o aumento na resistência à insulina em resposta à hipóxia intermitente prolongada era dependente da ruptura das vias de leptina.

Conforme esperado, os indivíduos com lipoproteína de High Density Lipoproteincholesterol (HDL-c, Alta Densidade-colesterol) elevado apresentaram menores valores de $\mathrm{IAH}$, porém o Low Density Lipoprotein-cholesterol (LDL-C, Lipoproteína de Baixa Densidadecolesterol) e CT não se elevaram proporcionalmente ao grau da apneia. Em protocolos experimentais, Savransky et al. ${ }^{44}$ observaram que nove em cada 10 ratos expostos à hipóxia intermitente com uma dieta rica em colesterol desenvolveram lesões ateroscleróticas, juntamente com aumentos significativos nos níveis de colesterol total e LDL-c e uma diminuição no HDL-c. Outro grupo de pesquisa estudou 255 indivíduos adultos de uma comunidade chinesa em Hong Kong, de ambos os sexos e com idades entre 30 e 60 anos, não tendo sido identificada associação entre a SAOS e os níveis de HDL-c e TG ${ }^{5}$. Os autores explicam que a apneia do sono pode influenciar os níveis circulantes de lipídeos ou apenas modular a função deles, o poderia aumentar o risco de doença cardiovascular nos indivíduos acometidos pela SAOS.

Foram observadas maiores diferenças com significância estatística das medidas antropométricas entre os grupos SAOS leve e grave. Contudo, não houve diferença em relação à significância estatística entre os grupos SAOS leve e moderado, mesmo que as médias de todos os valores estivessem aumentadas quando analisadas em associação com o grau da SAOS. Resultados semelhantes foram encontrados por Lovin et al. ${ }^{5}$, que observaram que a média do IMC e da idade foram maiores no grupo SAOS grave, quando comparado aos grupos SAOS leve e moderada, mas as diferenças não apresentaram significância estatística ( $p>0,05)$. O \%GC, a CA e a $C P$ foram significativamente maiores no grupo SAOS grave em relação ao leve $(p<0,01)$. A CC e os compartimentos de composição corporal também foram diferentes entre os grupos SAOS grave e moderada $(p<0,05)$. A CP foi significativamente diferente entre os grupos SAOS moderada e leve $(p<0,05)$. A obesidade geral e, principalmente, a abdominal contribuíram como principais fatores determinantes da hipoventilação e do acúmulo de gordura na região do pescoço, o qual foi apontado como preditor para o surgimento da SAOS.

O DAS apresentou moderada correlação com o IAH em relação ao \% GC, IMC, CC e a CP. A maioria das evidências existentes mostram resultados opostos, o que indica relação entre a gordura corporal e a gravidade da SAOS ${ }^{9,45}$. Lee \& Gallagher ${ }^{9}$ constataram que o percentual de gordura corporal estimado por bioimpedância previu o risco para SAOS, de modo a contribuir para probabilidade pré-teste.

Já no estudo de Katz et al. ${ }^{46}$, a aferição da medida da CP de 123 pacientes com ronco frequente revelou que os portadores de SAOS tinham pescoços mais espessos. Segundo esses autores, dentre IMC, idade, circunferência interna da faringe e do pescoço, a CP foi identificada como o mais importante preditor da SAOS. Correlações similares foram observadas por Oðretmenoðlu et al. ${ }^{45}$ no que diz respeito à $C P$ apresentar coeficientes de correlação mais fracos que o IMC e a CC. Entretanto, contrariamente aos dados desse estudo, o presente trabalho mostrou moderada correlação quanto ao DAS. Em virtude da escassez de dados na literatura sobre a associação do DAS com a SAOS, não foi possível conduzir uma discussão mais detalhada. Por outro lado, a utilização dessa medida antropométrica possui melhor associação com a detecção da gordura visceral, uma vez que a gordura subcutânea se espalha na posição supina e permite maior exposição do compartimento visceral. Logo, permite a mensuração do risco metabólico e, assim, da SAOS. Outros autores ${ }^{5,6,13}$ confirmaram esses resultados, os quais sugerem que as medidas 
antropométricas utilizadas no presente estudo e o perfil metabólico podem ser fatores preditores na determinação da gravidade da SAOS.

Dentre as limitações aqui encontradas, ressalta-se o número relativamente pequeno da amostra. Grande parte dos participantes era de meia-idade e já tinham sido encaminhados ao estudo do sono para polissonografia. Dessa forma, os resultados encontrados podem não ser aplicáveis a outros grupos etários, o que dificulta a avaliação do grupo sem a SAOS constituído por menor valor amostral. Outro fator limitador importante foi a impossibilidade de diferenciação da gordura visceral e da subcutânea, devido a limitações de equipamentos para aferição. Dentre eles, a tomografia computadorizada ${ }^{47}$, a densitometria por dupla emissão de Raios-X (DEXA) ${ }^{48}$ e a ressonância magnética ${ }^{49}$ têm sido utilizadas com maior acurácia na avaliação da composição corporal em pacientes com SAOS. Por fim, a natureza transversal do estudo não prova as relações de causa e efeito entre a SAOS e os perfis metabólico e nutricional dos indivíduos estudados.

\section{O N CLUS Ã O}

A relação entre a obesidade e a SAOS já é conhecida na literatura, o que evidencia a necessidade da definição de parâmetros antropométricos e clínicos viáveis e acessíveis para a detecção da doença. O estudo sugere o uso do DAS como bom preditor de risco para SAOS, por apresentar melhor associação com a gravidade da síndrome e ser capaz de subsidiar seu prognóstico. Esse achado merece destaque, já que o DAS é visto como uma boa ferramenta para mensurar a gordura visceral.

\section{COLABORADORES}

CMAT SÁ, RP PEDROSA e AC CAVALCANTI participaram na elaboração de estratégia experimental. MF LUSTOSA, RAB MEDEIROS e LPV NOVA participaram da coleta de dados, tabulação e discussão dos resultados e elaboração do artigo.

\section{REFERÊ NCIAS}

1. American Academy of Sleep Medicine. International classification of sleep disorders Diagnostic and coding manual. $2^{\text {nd }}$ ed. Westchester: American Academy of Sleep Medicine; 2005.

2. Punjabi NM. The epidemiology of adult obstructive sleep apnea. Proc Am Thorac Soc. 2008; 5(2):136-43. http://dx.doi.org/10.1513/pats.200709-155MG

3. Logan AG, Perlikowski SM, Mente A, Tisler A, Tkacova $R$, Niroumand $M$, et al. High prevalence of unrecognized sleep apnoea in drug-resistant hypertension. J Hypertens. 2001; 19(12):2271-7.

4. Pedrosa RP, Lorenzi-Filho G, Drager LF. Síndrome da apnéia obstrutiva do sono e doença cardiovascular. Rev Med. 2008; 87(2):121-7. http://dx.doi. org/10.11606/issn.1679-9836.v87i2p121-127

5. Lovin S, Bercea R, Cojocaru C, Rusu G, Mihaescu T. Body composition in obstructive sleep apneahypopnea syndrome bio-impedance reflects the severity of sleep apnea. Multidiscip Respir Med. 2010; 5(1):44-9. http://dx.doi.org/10.1186/20496958-5-1-44

6. Lubrano C, Saponara M, Barbaro G, Specchia P, Addessi $E$, Costantini $D$, et al. Relationships between body fat distribution, epicardial fat and obstructive sleep apnea in obese patients with and without metabolic syndrome. Plos One. 2012; 7(10):1-8. http://dx.doi.org/10.1371/journal.pone. 0047059

7. Vasques ACJ, Priore SE, Rosado LEFPL, Franceschini SCC. Utilização de medidas antropométricas para a avaliação do acúmulo de gordura visceral. Rev Nutr. 2010; 23(1):107-18. http://dx.doi.org/10.15 90/S1415-52732010000100012

8. Schafer H, Pauleit D, Sudhop T, Gouni-Berthold I, Ewig S, Berthold HK. Body fat distribution, serum leptin, and cardiovascular risk factors in men with obstructive sleep apnea. Chest. 2002; 122(3):829-39. http://dx.doi.org/10.1378/chest.122.3.829

9. Lee SY, Gallagher D. Assessment methods in human body composition. Curr Opin Clin Nutr Metab Care. 2008; 11(5):566-72. http://dx.doi.org/10.1097/ MCO.0b013e32830b5f23

10. Schipper MH, Jellema K, Rijsman RM. Occurrence of obstructive sleep apnea syndrome in patients with transient ischemic attack. J Stroke Cerebrovasc Dis. 2016; 25(5):1249-53. http://dx.doi.org/ 10.1016/j.jstrokecerebrovasdis.2016.01.046

11. Frija-Orvoën E. Syndrome d'apnées obstructives du sommeil: Complications métaboliques. Rev Mal Respir. 2016; 33(6):474-83. http://dx.doi.org/ 10.1016/j.rmr.2015.11.014 
12. Drager LF, Togeiro SM, Polotsky VY, Lorenzi-Filho G. Obstructive sleep apnea: A cardiometabolic risk in obesity and the metabolic syndrome. J Am Coll Cardiol. 2013; 62(7):569-76. http://dx.doi.org/10. 1016/j.jacc.2013.05.045

13. Tasali E, Ip MSM. Obstructive sleep apnea and metabolic syndrome: Alterations in glucose metabolism and inflammation. Proc Am Thorac Soc. 2008; 5(2):207-17. http://dx.doi.org/10.1513/pats. 200708-139MG

14. Nock NL, Li L, Larkin EK, Patel SR, Redline S. Empirical evidence for "syndrome Z": A hierarchical 5-factor model of the metabolic syndrome incorporating sleep disturbance measures. Sleep. 2009; 32(5):615-22.

15. American College of Sports Medicine. Guidelines for exercise testing and prescriptions. $4^{\text {th }}$ ed. Philadelphia: Lea and Febiger; 1991.

16. Ferreira MG, Valente JG, Gonçalves-Silva RMV, Sichieri R. Consumo de bebida alcoólica e adiposidade abdominal em doadores de sangue. Rev Saúde Pública. 2008; 42(6):1067-73. http://dx. doi.org/10.1590/S0034-89102008000600013

17. World Health Organization. Obesity: Preventing and managing the global epidemic. Report of a WHO Consultation. Geneva: WHO; 2000.

18. Waitzberg DL. Nutrição oral, enteral e parenteral na prática clínica. $4^{a}$ ed. São Paulo: Atheneu; 2009. v. 1 .

19. Chumlea WC, Roche AF, Steinbaugh ML. Estimating stature from knee height for persons 60 to 90 years of age. J Am Geriatr Soc. 1985; 33(2):116-20. http://dx.doi.org/10.1111/j.1532-5415.1985. tb02276.x

20. Cuppari L, Schor N. Guia de nutrição: nutrição clínica do adulto. $2^{a}$ ed. São Paulo: Manole; 2005.

21. Organización Panamericana de la Salud. Anales $36^{\circ}$ Reunón del Comitê Acesor de Investigaciones em Salud - Encuestra Multicêntrica - Salud Bienestar y Envejecimeiento (SABE) en América Latica e el Caribe - Informe preliminar, Washington: OPAS; 2002.

22. Valdez R. A simple model-based index of abdominal adiposity. J Clin Epidemiol. 1991; 44(9):955-6. http://dx.doi.org/10.1016/0895-4356(91)90059-i

23. Fitch KV, Stanley TL, Looby SE, Rope AM, Grinspoon SK. Relationship between neck circumference and cardiometabolic parameters in HIV-infected and non-HIV-infected adults. Diabetes Care. 2011; 34(4):1026-31. http://dx.doi.org/10.2337/dc10-19 83

24. Sociedade Brasileira de Cardiologia. V Diretriz Brasileira de Dislipidemias e Prevenção da Ateros- clerose Diretrizes. Arq Bras Cardiol. Rio de Janeiro, 2013; 101(4):1-22.

25. Flemons WW, Buysse D, Redline S, Strohl K, Wheatley J, Douglas N, et al. Sleep-related breathing disorders in adults: Recommendations for syndrome definition and measurement techniques in clinical research: The Report of an American Academy of Sleep Medicine Task Force. Sleep. 1999; 22(5):667-89.

26. Friedewald WT, Levy RL, Fredrickson DS. Estimation of the concentration of low-density lipoprotein cholesterol in plasma without the use of preparative ultracentrifuge. Clin Chem. 1972, 18(6):499-502.

27. Sociedade Brasileira de Diabetes. Diretrizes da Sociedade Brasileira de Diabetes. $3^{\mathrm{a}}$ ed. Itapevi: A. Araújo Silva Farmacêutica; 2009.

28. Franklin SS, Jacobs MJ, Wong ND, L'Italien GJ, Lapuerta P. Pre-dominance of isolated systolic hypertension among middle-aged and elderly US hypertensives: Analysis based on national health and nutrition examination Survey (NHANES) III. Hypertension. 2001; 37:869-74. http://dx.doi.org/ 10.1161/01.HYP.37.3.869

29. Shimura R, Tatsumi K, Nakamura A, Kasahara Y, Tanabe N, Takiguchi $Y$, et al. Fat accumulation, leptin, and hypercapnia in obstructive sleep apneahypopnea syndrome. Chest. 2005; 127(2):543-9. http://dx.doi.org/10.1378/chest.127.2.543

30. Young T, Peppard PE, Gottlieb DJ. Epidemiology of obstructive sleep apnea: A population health perspective. Am J Respir Crit Care Med. 2002; 165(9):1217-39. http://dx.doi.org/10.1164/rccm. 2109080

31. Chang $\mathrm{E}$, Wang $\mathrm{H}$. Gender differences in obstructive sleep apnea syndrome. Eur Resp J. 2015; 46(59):2363. http://dx.doi.org/10.1183/13993 003.congress-2015.PA2363

32. Assal HH, Kamal E. Gender differences in polysomnographic findings in Egyptian patients with obstructive sleep apnea syndrome, Egypt. J Chest Dis Tuberc. 2016; 65(3):649-54. http:// dx.doi.org/10.1016/j.ejcdt.2016.03.009

33. Malhotra A, Huang Y, Fogel RB, Pillar G, Edwards $J K$, Kikinis $R$, et al. The male predisposition to pharyngeal collapse: Importance of airway length. Am J Respir Crit Care Med. 2002; 166(10):1388-95. http://dx.doi.org/10.1164/rccm.2112072

34. Kritikou I, Basta M, Tappouni R, Pejovic S, Fernandez-Mendoza J, Nazir R, et al. Sleep apnoea and visceral adiposity in middle-aged male and female subjects. Eur Respir J. 2013; 41:601-9. http:// dx.doi.org/10.1183/09031936.00183411

35. Trémollieres FA, Pouilles JM, Ribot CA. Relative influence of age and menopause on total and 
regional body composition changes in postmenopausal women. Am J Obstet Gynecol. 1996; 175(6):1594-600. http://dx.doi.org/10.1016/ S0002-9378(96)70111-4

36. Dancey DR, Hanly PJ, Soong C, Hoffstein V. Impact of menopause on the prevalence and severity of sleep apnea. Chest. 2001; 120(1):151-5. http://dx. doi.org/10.1378/chest.120.1.151

37. Polotsky M, Elsayed-Ahmed AS, Pichard L, Harris CC, Smith PL, Schneider H, et al. Effects of leptin and obesity on the upper airway function. J Appl Physiol. 2012; 112(10):1637-43. http://dx.doi.org/ 10.1152/japplphysiol.01222.2011

38. Cuhadaroðlu C, Utkusava` A, Oztürk L, Salman S, Ece T. Effects of nasal CPAP treatment on insulin resistance, lipid profile, and plasma leptin in sleep apnea. Lung. 2009; 187(2):75-81. http://dx.doi. org/10.1007/s00408-008-9131-5

39. Bhama JK, Spagnolo S, Alexander EP, Greenberg M, Trachiotis GD. Coronary revascularization in patients with obstructive sleep apnea syndrome. Heart Surg Forum. 2006; 9(6):813-17. http://dx.doi. org/10.1532/HSF98.20061072

40. Nakagawa Y, Kishida K, Kihara S, Yoshida R, Funahashi T, Shimomura I. Nocturnal falls of adiponectin levels in sleep apnea with abdominal obesity and impact of hypoxia-induced dysregulated adiponectin production in obese murine mesenteric adipose tissue. J Atheroscler Thromb. 2011; 18(3):240-7. http://dx.doi.org/10. 5551/jat.6593

41. Lopes WA, Leite N, Silva LR, Consentino CLM, Coutinho P, Radominski RB, et al. Comparação de três equações para predição da gordura corporal por bioimpedância em jovens obesos. Rev Bras Med Esporte. 2015; 21(4):266-70. http://dx.doi.org/10. 1590/1517-869220152104146763

42. Hoffstein V, Mateika S. Differences in abdominal and neck circumferences in patients with and without obstructive sleep apnoea. Eur Respir J. 1992; 5(4):377-81.
43. Polotsky VY, Li J, Punjabi NM, Rubin AE, Smith PL, Schwartz AR, et al. Intermittent hypoxia increases insulin resistance in genetically obese mice. J Physiol. 2003; 552(1):253-64. http://dx.doi.org/10.1113/ jphysiol.2003.048173

44. Savransky V, Nanayakkara A, Li J, Bevans S, Smith PL, Rodriguez A, et al. Chronic intermittent hypoxia induces atherosclerosis. Am J Respir Crit Care Med. 2007; 175(12):1290-7. http://dx.doi.org/10.1164/ rccm.201209-16880C

45. Oðretmenoðlu O, Süslü AE, Yücel OT, Onerci TM, Sahin A. Body fat composition: A predictive factor for obstructive sleep apnea. Laryngoscope. 2005; 115(8):1493-8. http://dx.doi.org/10.1097/01.mlg. $0000172204.82314 . c 3$

46. Katz I, Stradling J, Slutsky AS, Zamel N, Hoffstein $\mathrm{V}$. Do patients with obstructive sleep apnea have thick necks? Can Am Rev Respir Dis. 1990; 141(5):1228-31. http://dx.doi.org/10.1164/ajrccm/ 141.5_Pt_1.1228

47. Yu JH, Ahn JH, Yoo HJ, Seo JA, Kim SG, Choi KM, et al. Obstructive sleep apnea with excessive daytime sleepiness is associated with non-alcoholic fatty liver disease regardless of visceral fat. Korean J Intern Med. 2015; 30(6):846-55. http://dx.doi.org/ 10.3904/kjim.2015.30.6.846.

48. Bhatia R, Lesser DJ, Oliveira FG, Tran WH, Keens TG, Khoo MC, et al. Body fat composition: A predictive factor for sleep related breathing disorder in obese children. J Clin Sleep Med. 2015; 11(9):1039-45. http://dx.doi.org/10.5664/jcsm. 5022

49. Degache F, Sforza E, Dauphinot V, Celle S, Garcin $A$, Collet $\mathrm{P}$, et al. Relation of central fat mass to obstructive sleep apnea in the elderly. Sleep. 2013; 36(4):501-7. http://dx.doi.org/10.5665/sleep.2532

Recebido: fevereiro 5, 2016 Versão final: junho 13, 2016 Aprovado: junho 30, 2016 